lesion showing characters of osteitis fibrosa and osteomalacia. Unnecessary PTX or even harmful PTX should be avoided strictly.

Use of desferroxamine (DFO); DFO was originally used to remove iron from tissues by chelation. Application of this agent to Al-related osteomalacia has brought a new therapeutic benefit in past three years. In fact, use of DFO during dialysis decreases bone and joint pain, or muscle weakness. The effect of DFO is clearly demonstrated by measuring serum levels of $\mathrm{Al}$ or diminishing $\mathrm{Al}$ accumulation in bone tissue with clinical improvement.

Kidney transplantation: Although the prevention of steroid induced bone disease or hypophosphatemic osteomalacia caused by excess loss of phosphate following successful transplantation still remains unresolved, kidney transplantation, at this time, may offer the only adequate therapy of pathologic distrubances induced by loss of kidney function such as not only excretory but also endocrine function.

In summary, the understanding of pathophysiological mechanisms of dialysis bone disease and clinical use of active vitamin $\mathrm{D}$ metabolites have brought remarkable improvement of ROD, nevertheless, there are many cases which do not respond to adequate treatment. This fact means that metabolic metabolic abnormalities of ROD is extremely complex.

Thus, further fundamental investigation on bone metabolism, parathyroid endocrinology, vitamin $\mathrm{D}$ endocrinology and intestinal transport of minerals will be required in addition of accumulation of clinical observations.

\title{
(5) Body Fluid Homeostasis
}

\author{
Eiichi KATO and Takao WADA
}

Department of Internal Medicine, School of Medicine, Keio University, Tokyo

\section{Introduction}

Homeostasis has been an important subject for medical scientists, ever since this term was coined by Cannon in 1932. Sixteen years ago, one of the authors, Kato, made a speech about the same subject at the symposium of this meeting. Since then much advancement occurred in the field of medicine as well as in others. Especially, the recent progress of systems control theory enabled us to develop an entirely new method of describing the homeostatic processes in the body, which will be presented in the present speech, along with other basic aspects of the body fluid physiology in the patients with chronic renal failure.

\section{Homeostasis and "Homeostenosis"}

In a word, the progression of chornic renal failure can be expressed as the process of decrease in the nephron population in the diseased kidney. Although the remaining nephrons fully work to maintain the homeostasis, the decline in the overall kidney function cannot help leading the body to the state of "homeostenosis" rather than homeostasis.

Although various biochemical abnormalities are produced by the decompensation thus occurring, the threshold for each of those is variable depending on the degree of development of regulatory systems for that particular clinical parameter. The mode of increase in some substances, such as serum creatinine, which is almost free from regulation, straightly reflects the decline in the kidney function. However, main electrolytes, playing important roles to maintain body fluid homeostasis, more or less show some delay of the appearance of their abnormalities when compared with that of the abnormalities of creatinine.

It seems as if the kidney endeavors to exert its maximal function remaining for maintaining electrolyte balances. Let us assume that the normal GFR of $100 \mathrm{ml} / \mathrm{min}$ goes down to $1 / 10$ of it, i.e. $10 \mathrm{ml} / \mathrm{min}$. On the diet containing $15 \mathrm{~g} /$ day of $\mathrm{NaCl}$, in order to maintain the in-out balance of 
sodium, the kidney is expected to suppress sodium reabsorption from the normal value of $99 \%$ down to $88 \%$ at that time. In the case of potassium, the kidney should increase its secretion to its extreme degree, since the excreted fraction of potassium is expected to rise from the normal value of $10 \%$ even to $100 \%$. This pathological homeostasis should continue until some therapeutic intervention, such as hemodialysis or kidney transplantation.

\section{Spectral Analysis and Homeostasis}

Spectral analysis enables us to decompose a stochastic process into the spectrum of sinusoidal components each with a single frequency. In spite of the usefulness of this analytical method, its limitation in the analysis of a feedback system was pointed out and a practical method for this purpose was developed by Akaike by using multivariate auto-regressive models in 1967.

We introduced this approach to spectral analysis for analyzing the process of homeostasis in the body. The two main concepts thus adopted were Akaike's relative power contribution (ARPC) and impulse response function. The former expresses the degree of contribution of the fluctuation of a certain variable to that of another. This concept is completely different from the conventional "degree of contribution" calculated from correlation coefficients, since the latter statistical parameter expresses only the degree of parallelism between two variables. Accordingly, even if there is some correlation between $\mathrm{X}$ and $\mathrm{Y}$, one cannot say which contributes to which. In contrast, ARPC can positively tell which contributes to which, especially because it includes the concept of time lapse in itself.

Figure 1 shows various factors contributing to fluctuation of blood pressure in a hemodialysis patient. The abscissa is the scale for the frequency, expressed as cycle/week, of the sinusoidal components obtained by decomposing the fluctuation of variables analyzed. The ordinate is the scale for ARPC representing the rate of contribution of the various factors to the fluctuation of blood pressure. It can be seen that the fluctuations of plasma renin activity (PRA), plasma bicarbonate, and body weight much contributed to the fluctuation of blood pressure especially at the lower frequency

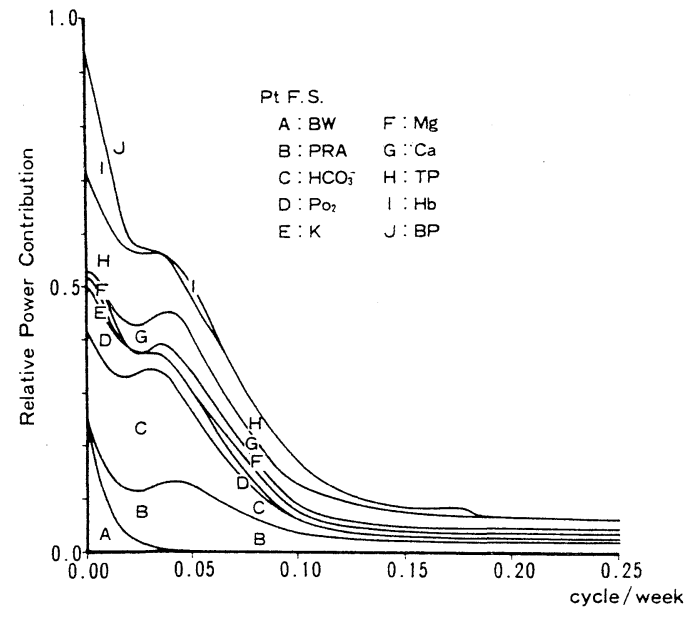

Fig. 1. Various factors contributing to fluctuation of blood pressure in a HD patient.

range. According to our experiences, the lower frequencies of less than 0.1 cycle/week are closely related to the metabolic networks in the body. It is apparent that other factors more closely related to higher frequencies also contribute to more rapid fluctuation of blood pressure in hypertensive patients. Actually, most clinicians notice that the nervous tension causes a very transient rise in blood pressure. Therefore, each of the causal factors for hypertension may be related with a particular frequency range. Such a frequency dependency was demonstrated in our previous studies ${ }^{1,2)}$. Anyway these findings seem to validate the mosaic hypothesis for hypertension proposed by Page, on the condition that the hypothesis is somewhat modified by taking the concept of frequency domain into consideration.

Although ARPC is much helpful for elucidating the network relationships among the variables under study, it does not show the actual movements of those variables which are triggered by the fluctuation of some of them. Such movements can be demonstrated by the use of impulse response functions obtained from the autoregressive coefficients. Figure 2 shows the response to blood pressure triggered by the impulse of plasma bicarbonate, PRA, or body weight. Thus one can visualize the mode of homeostasis in the body.

\section{Conclusion}

It is apparent that the progress of medicine is much affected by that of various fields of science 


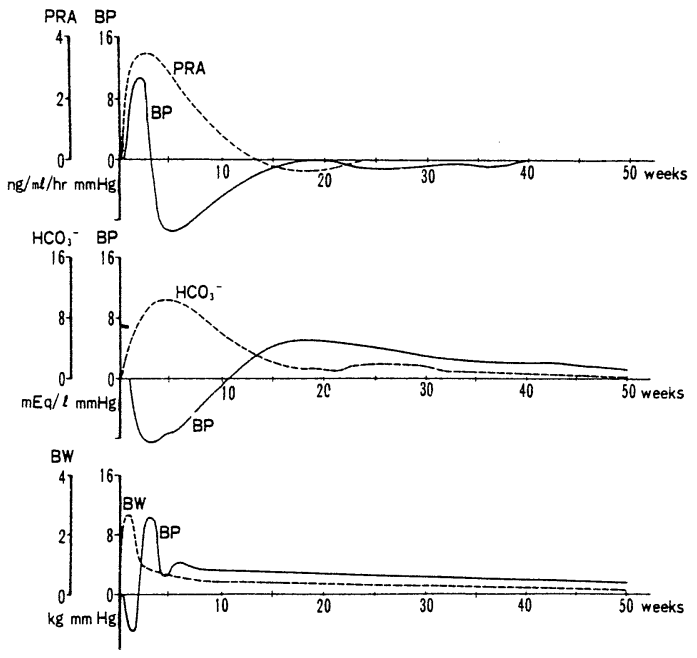

Fig. 2. Response of blood pressure to impulse of various factors. surrounding it. The knowledge about the homeostasis cannot be an exception in this respect. Although this concept has been regarded by most clinicians as only an abstract entity, it has now changed to a concrete one. We believe that the approach presented in this study should become a powerful tool for unveiling the complicated networks in the body, including immunologic, endocrinologic and metabolic ones.

\section{REFERENCES}

1) Wada $\mathrm{T}$, Akaike $\mathrm{H}$ and Kato $\mathrm{E}$ : Autoregressive models provide stochastic descriptions of homeostatic processes in the body. Jap J Nephrol 28: 263, 1986.

2) Wada T, Kato E, et al: Frequency dependency of causal factors for hypertension in hemodialysis patients. Jap J Nephrol (in press).

\title{
3. The Role of Dietary Treatment in the Conservative Management of Chronic Renal Failure
}

\author{
Kiyofumi Hirata
}

Department of Nephrology, Toho University School of Medicine

\begin{abstract}
Although it has long been believed that progression of chronic renal failure (CRF) cannot be ceased, recent advances in the treatment of chronic renal disease provide evidence of slowing proggression of CRF by means of dietary approach$\mathrm{es}^{1-6)}$.

This paper is to study the beneficial effects of dietary treatment in patients with CRF and discuss the role of diet in the conservative management of CRF.
\end{abstract}

\section{PATIENTS AND METHODS}

99 patients with CRF were classified into three groups. Group 1 consisted of 50 patients who started with diet, but eventually underwent dialysis treatment in an average 14.1 months. Group 2 comprised 32 patients who continued diet for a mean period of 23.8 months. Group 3 was 17 patients selected from those who has been on diet over the past $4-11$ years (mean 77.8 months)
(Table 1). Distributions of age, sex and underlying diseases were comparable between these three groups. The diet contained daily $20 \mathrm{~g}$ or $30 \mathrm{~g}$ protein of high biological values and at least $35 \mathrm{kcal}$ per kg per day.

Determinations for blood urea nitrogen (BUN), serum creatinine (Scr), serum total protein (TP), hematocrit ( $\mathrm{Ht}$ ), serum calcium (Sca) and serum phosphorus $(\mathrm{Sp})$ were done before and after the dietary treatment. The pre- and pottreatment data (mean $\pm 1 \mathrm{SD}$ ) were compared by paired Student's t-test, and the slopes of linear regression lines obtained both pre- and posttreatment compared by Snedecor and Cochran's methods ${ }^{7)}$.

\section{RESULTS}

Table 1 shows the results obtained before and after dietary treatment in each group.

BUN level in group 1 or in group 2 significantly increased or decreased after diet, respectively, 